\title{
AN EXPLORATORY STUDY OF THE MEALTIME EXPERIENCE OF OLDER PEOPLE WITH DYSPHAGIA
}

\author{
S. Ullrich ${ }^{1}$ J. Buckley ${ }^{2}$, J. Crichton ${ }^{3}$, A. Esterman ${ }^{4}$
}

\begin{abstract}
People with dysphagia are at high risk of malnutrition. To maintain safe oral intake, solid food may be texture modified but this is associated with a reduction in the enjoyment of the eating experience. A recent approach to improving the enjoyment of eating texture-modified food has been to mould the food into the shape of the food that has been modified. The aim of this exploratory study was to describe and explain the mealtime experience of older people with dysphagia. Design: Qualitative and exploratory. Participants: In total, thirty five participants (nursing, care workers, lifestyle assistants, catering staff and residents) were involved in non-participant observations and individual interviews for the qualitative assessment of the eating experience. Intervention: Moulded texture-modified food. The intervention occurred at lunchtime for a period of 3 consecutive days. Qualitative assessment: Non-participant observations and individual interviews were conducted before and after the intervention. Analysis: Interpretative, descriptive and explanatory. Findings: Residents with dysphagia are separated from the dining experience and fostering good relationships between residents at mealtimes may lessen the effects of the challenging eating behaviours that often isolate residents with dysphagia from the dining environment. Non-moulded texture-modified food was viewed negatively by all participants and contributed to problems of interaction between care staff and residents by weakening those conversations and interactions that underpin the social dining experience. Residents also experienced difficulties adjusting to texture-modified food. Conclusion: Residents' experience of adjustment to texture-modified food is difficult and non-moulded texture-modified meal that is unrecognisable and indescribable creates a problem of interaction between care staff and residents during mealtimes. The implementation of moulded texture-modified food has resulted in positive qualitative outcomes in the perception of texturemodified food and has improved the verbal interaction between care staff and residents.
\end{abstract}

Key words: Texture-modified food, elderly, dysphagia, aged care, exploratory.

\section{Introduction}

Malnutrition remains a recognised problem within residential aged care facilities. Age-related changes and the catabolic effects of acute or chronic diseases place older people at high risk of malnutrition (1). An international review of the prevalence of malnutrition across residential aged care facilities suggests that between 12 percent and 85 percent of residents are malnourished, with up to 100 percent of residents at nutritional risk (1). There is a lack of information on the prevalence of malnutrition in Australian residential aged care facilities. However, a recent study reported the

1. University of South Australia Adelaide, South Australia; 2. Medical Statistics, Chair of Biostatistics Sansom Institute of Health Service Research and School of Nursing and Midwifer; 3. Nutritional Physiology Research Centre Sansom Institute for Health Research University of South Australia; 4. Program Director School of Communication, international Studies and Languages University of South Australia.

Corresponding Author: Sandra Ullrich, Research/ Consultant, University of Sout Australia, Nursing and Midwifery, GPO BOX 2471, Adelaide, South Australia 5001, Australia, +61883022749, work: +61882911079, FAX: +6182911085, Sandra.Ullrich@ unisa.edu.au

prevalence of malnutrition in a number of Queensland residential aged care facilities to be at 50 percent (2). Furthermore, an investigation into nutritional risk in one residential care facility in Victoria found that 11 percent of residents were underweight and 68 percent had low levels of at least one serum marker, indicating that nearly 75 percent may have been at risk of nutrition related diseases (3).

People with dysphagia are at high risk of malnutrition. Dysphagia is defined as difficulty with swallowing; specifically it refers to a sensation causing one to perceive impairment in the passage of food from mouth to stomach (4). Up to $60 \%$ of residents in residential aged care facilities experience dysphagia (4). Dysphagia is a common condition, especially among people with disability and those of increasing age. Improving the delivery of nutritional care to older people with dysphagia will be an increasingly important healthcare issue because of the ageing population (5).

Dysphagia is an important safety concern for health care providers because it affects eating habits by causing anxiety or panic during mealtimes and leads 
to feelings of social isolation and depression (6-8). To maintain safe oral intake, solid food may be texturally modified according to The Australian Standards for Texture Modified Food and Fluids (5). Consequently, texture-modified foods are rarely a diet of choice, but a diet of necessity for individuals with dysphagia if they are to maintain their nutritional needs orally (5). Whilst texture-modification of food decreases consumer acceptance of the meal and diminishes nutritional intake (9-12), moulded texture-modified food has been shown to increase the nutrient intake and body weight of older people with dysphagia (13). However, the association between texture-modified food and the concept of nutritional 'necessity' has resulted in little research in this area and the identification of a number of knowledge gaps related to standardised production, inappropriate use of texture-modified food, the nutritional quality and low acceptability of texture-modified food $(13,8)$. Although the issues associated with the use of texturemodified foods has become increasingly recognised (8), few studies exist which explore, describe and explain the mealtime experience of older people with dysphagia.

\section{Design, methods and setting}

The study was conducted by the University of South Australia, in collaboration with Medirest Australia which provides specialist food, hospitality and support services to some hospitals and seniors living in residential aged care facilities throughout Australia, and Eldercare which is a large aged care provider currently offering approximately 1000 licensed aged care beds across 12 sites in metropolitan Adelaide and the Yorke Peninsula in South Australia. The study was conducted across two (i.e. Site A and Site B) of Eldercare's aged care sites. The study was approved the University of South Australia's Human Research Ethics Committee in 2013. In order to participate in the study all participants were asked to provide written consent.

\section{Design}

This exploratory study seeks to find how older people with dysphagia experience mealtimes within a residential aged care context and what concerns them. This study is also explanatory as it seeks to describe the effects of moulded texture-modified food on the mealtime experience of older people with dysphagia (14). The following questions will be explored:

1. What factors that influence the delivery of texturemodified food to older people with dysphagia.

2. How does moulded texture-modified food enhance the mealtime experience of older people with dysphagia compared to non-moulded texture-modified food?

3. Why does moulded texture-modified food enhance the mealtime experience of older people with dysphagia compared to non-moulded texture-modified food?
Non-moulded texture-modified food refers to pureed food that is shapeless and does not resemble the food that it represented prior to its modification. Moulded texturemodified food refers to pureed food that is formed into the shape of the food (e.g. broccoli, carrots, peas, fish etc...) that it represented prior to its modification. It is shaped using durable food grade polypropylene moulds.

For this study, the moulded texture-modified food was not flavour enhanced or coloured. The intervention occurred at lunchtime for a period of 3 consecutive days. All participants received the same menu cycle (i.e. a series of menus planned for a particular period of time) and the texture was adjusted for all residents, when needed, with the assistance of a dietician.

\section{Participant inclusion and exclusion criteria}

Thirty-five participants (nursing, care staff, catering staff and residents) were involved in the non-participant observations and individual interviews were conducted with twenty-nine care staff (nursing, care workers, lifestyle assistants and catering staff) and 6 residents. All participants were screened according to the following inclusion or exclusion criteria:

\section{Inclusion criteria}

1. Residents aged between 65 and 95 years of age with dysphagia which is defined as;

'a condition, a disorder or a symptom that may be genetic, developmental, acquired, functional or iatrogenic in origin. It can be caused by structural, physiological and / or neurological impairments affecting one or more stages of swallowing, namely the preparatory, oral, pharyngeal, and / or oesophageal stages. This may present as a difficulty with sucking, drinking, eating, controlling saliva, protecting the airway or swallowing (1).'

2. Residents able to provide informed consent (or consent was provided by residents' next-of-kin/legal representative).

3. Care staff (i.e. nurses, care workers and catering staff) able to provide informed consent.

\section{Exclusion criteria}

1. Terminally ill residents receiving palliative care were excluded from the study.

\section{Methods}

Overt non-participant observations were collected, through field notes, by the primary researcher before and during the intervention of moulded texture-modified food. The observation schedule was informed by Spradley's (1980) Developmental Research Sequence 
to observational data collection (15). Non-participant observations provided information about the behaviours, actions, and interactions of the participants in order to understand complex situations. The primary researcher conducted non-participant observation which allowed for the identification of relevant questions for subsequent individual interviews (15). Semi- structured individual interviews were also conducted to provide descriptive data and explain the patterns and associations between experiences, behaviours and perspectives, and relevant characteristics of the study population. Data collection continued until data saturation was reached. The interview data was transcribed verbatim and were returned to participants for member checking (16, 17). Triangulation was used to check the integrity of, and extend the inferences drawn from the data (18). Data management was conducted using NVIVO 9 data management software (19).

Analysis of non-participant observation field notes and individual interview data was qualitative and conducted by the primary researcher, using the interpretative, descriptive and explanatory approach of the Analytic Hierarchy $(20,21)$. The Analytic Hierarchy is described in the form of conceptual scaffolding where the process of data management and analysis is non-linear across nine 'viewing platforms'. The application of the Analytic Hierarchy involved three distinct but interrelated processes: data management, descriptive accounts and explanatory accounts. Data management involved identifying initial concepts and labelling the data by way of indexing. Data with similar properties were located together and associatively analysed by noting the concepts that weaved in and out of each other. The data associated with these central concepts were extracted as evidence for later representation. Descriptive accounts drew on the previous analysis and involved the exploration of data by detection, categorisation and classification with the aim of presenting explanatory accounts that are authentic, meaningful and provide content that is illuminating $(20,21)$.

\section{Findings}

\section{The dining environment}

Lunchtime commenced at 1200 at Site A and at 1230 at Site B. The architectural design of the mealtime settings were similar, however, aesthetically the environments varied considerably between sites. For example, wall partitions were moveable at Site A and enabled care staff to reconfigure the environment to accommodate a variety of meaningful resident activities, but remained fixed at the other. The mealtime environment was functionally orientated at Site A and presented limited dining cues for residents and care staff, whereas, the mealtime environment was domestically orientated at Site B and provided a number of important dining cues to residents and care staff (22). Dining cues included: the use of table cloths, napkins, placement of condiments on the table and a board to indicate the menu for the day.

Both mealtime environments presented similarities in terms of the clinical and non-clinical activities that occurred during resident mealtimes. The use of a mortar and pestle to crush medications occurred at both sites and medication trolleys were also prominently positioned within the dining rooms at mealtimes. Differences across sites included where care workers chose to cut up resident meals. At Site A, the cutting up of meals was done at the servery and at Site B this was done at the table in front of the resident and only after permission was sought from the resident. A Maître Dee (i.e. a care worker who oversaw the delivery of meals to residents) was found at Site B but not at Site A. White clothing protectors were placed on most residents at Site A, whereas blue checkered clothing protectors were placed on 3 residents at Site B, the rest being provided with white napkins.

Care staff, at both sites, stated that the mealtime environment was noisy, rushed and one care worker said that it felt like a 'production line'. The dining room had some inherent design challenges that included its large size, the inadequate arrangement of tables, the lack of space and the inappropriate use of wall partitions which had the unintended effect of denying residents who were seated in air-comfort chairs (also known as princess chairs which manage pressure problems and offers much better quality of life for the resident) access to their usual mealtime seating position.

Residents' (who consumed texture-modified food) seating arrangement at mealtimes varied across the two residential aged care sites. At Site A, seven residents were seated in air-comfort chairs, in a semi-circle, adjacent to the main dining area and not at dining tables; rather over-way tables were used to place the meal upon. At Site B, three residents (seated in air-comfort chairs) were positioned at tables in the main dining area.

\section{Residents' separation from the dining experience}

Care staff were aware of the separation of residents seated in air-comfort chairs from the main dining environment. Terms such as: 'them and us', segregation', 'isolation', 'outcasts' and being 'singled out' were used to describe these residents' experiences of mealtimes as shown in the following examples:

Care worker [5]: 'It's just very 'them and us' isn't it? Sitting in a circle watching each other eat.'

Care worker [1]: 'People that have the vitamised food are just like outcasts at the moment, sitting in a different area.'

A sense of empathy was found amongst care and catering staff about the seating arrangement of residents 
in air-comfort chairs:

Catering staff member [1]: 'I mean Jeff, I mean what is he thinking? He must be thinking 'Why am I over here and they're over there?"

Care worker [3]: 'I actually empathise, there's a lady downstairs that sits at a table with three other residents who have normal meals and she has to have vitamised.'

\section{Perceptions of non-moulded texture-modified food}

Interviews with care staff and residents found that opinions of the non-moulded texture-modified food were negative. These comments were primarily about the look of the food not appearing appetising:

Resident [1] husband: 'Oh she's getting sick of it being vitamised up like that. It doesn't look nice. I'll stay here for the evening meal most times and she'll look at it, but she doesn't like the look of it. It doesn't look very nice, but she's hungry so she eats it.'

Lifestyle assistant (engage residents in leisure and meaningful activities) [1]: ' $I$ 've seen a lot of residents look at this and roll their eyes and sort of go 'eugh'. Like 'why bother eating that then?"

Care worker [3]: 'That's Monday, Tuesday, Wednesday, Thursday, Friday, Saturday or Sunday.'

\section{Identification as guess work}

Non-participant observations and interviews with care staff and one resident found that 'guess work' was involved in an attempt to identify the non-moulded texture-modified food. Neither the care staff nor the residents were aware of what the food was that was being served. The following comments were typical:

Resident [5]: 'Well I mean I don't know what it is, I don't know whether that's carrots or mango or pumpkin.'

Care worker [9]: 'You go up to someone and I've had people say 'oh what's that?' and you're thinking pumpkin, carrot, sweet potato - god only knows and you just take a stab in the dark.'

\section{The non-moulded texture-modified meal and problems of interaction}

Non-participant observations found that there was a lack of interaction between care staff and residents when serving non-moulded texture-modified food. Care staff were also observed describing the non-moulded texture-modified food in nondescript deictic or generic terms such as 'this, that, it, lunch, dessert and sweets'. Care staff reported finding it difficult to describe the non-moulded texture-modified meal to residents and to engage with them socially at mealtimes:
Figure 1

Non-mounded texture-modified food

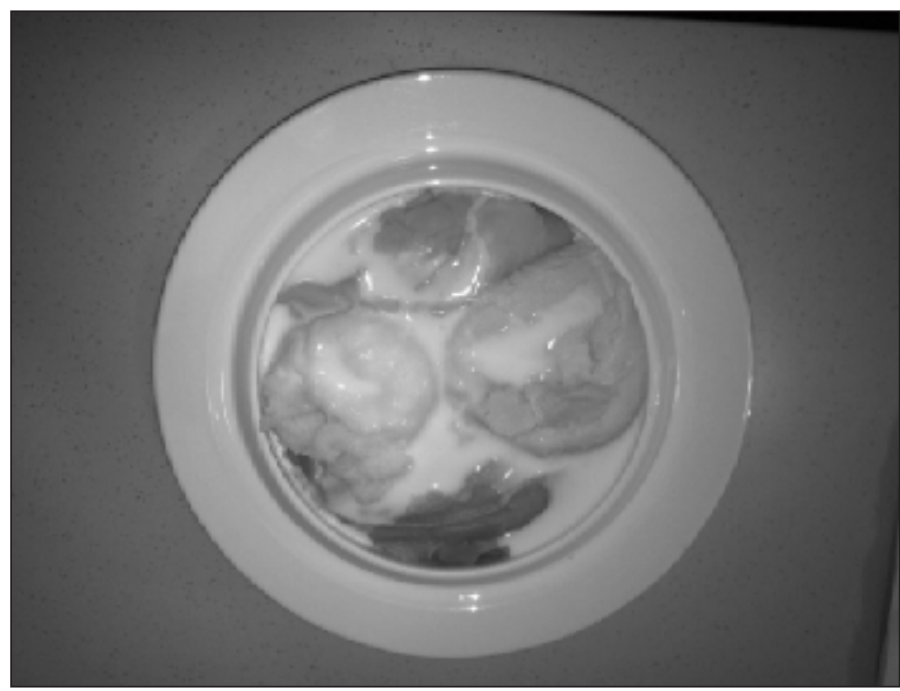

Care worker [5]: 'I guess they all look very similar, so I don't really know how to explain it to residents.'

Care worker [4]: 'It doesn't look like anything, it doesn't tell you anything and I don't know what it is, I can't see what it is.'

Lifestyle [2]: 'My conversation would be completely different. Reminiscing would be very challenging on the left hand side (non-moulded texture-modified food), but I would be reminiscing with the right side definitely (normal food).'

Care staff described a variety of negative feelings associated with serving non-moulded texture-modified food to residents. These included feeling unhappy, terrible and disgusted:

Care worker [6]: 'I can't begin to feel happy about serving it myself. How can I be happy about serving something like that if I wouldn't eat that myself?'

Care worker [4]: 'I feel terrible putting it in front of her.'

Care staff also described 'self-conscious' emotions when serving non-moulded texture-modified food to residents including emotions of guilt, dishonesty and embarrassment as shown in the following examples:

Care worker [4]: 'I probably feel guilty handing someone food that looks like that (non-moulded texturemodified food) and I mean there are some residents that I do apologise to and wish that it was better. I suppose it's more of a guilt thing.'

Care worker [9]: 'If they ask me what they were eating, then it gets tricky because it's obviously easier to explain to someone who is eating a normal diet what they're eating and how it looks scrumptious, but if you try and describe the blobs (non-moulded texture-modified food) to someone, you're like 'Oh look, that looks like you've got some yummy fish there' but it's just not sincere, you're trying, but you're just looking at it thinking 'that's just crap." 
Care worker [12]: 'When I take the vitamised diet around, I often feel quite embarrassed.'

Figure 2

Moulded texture-modified food (27)

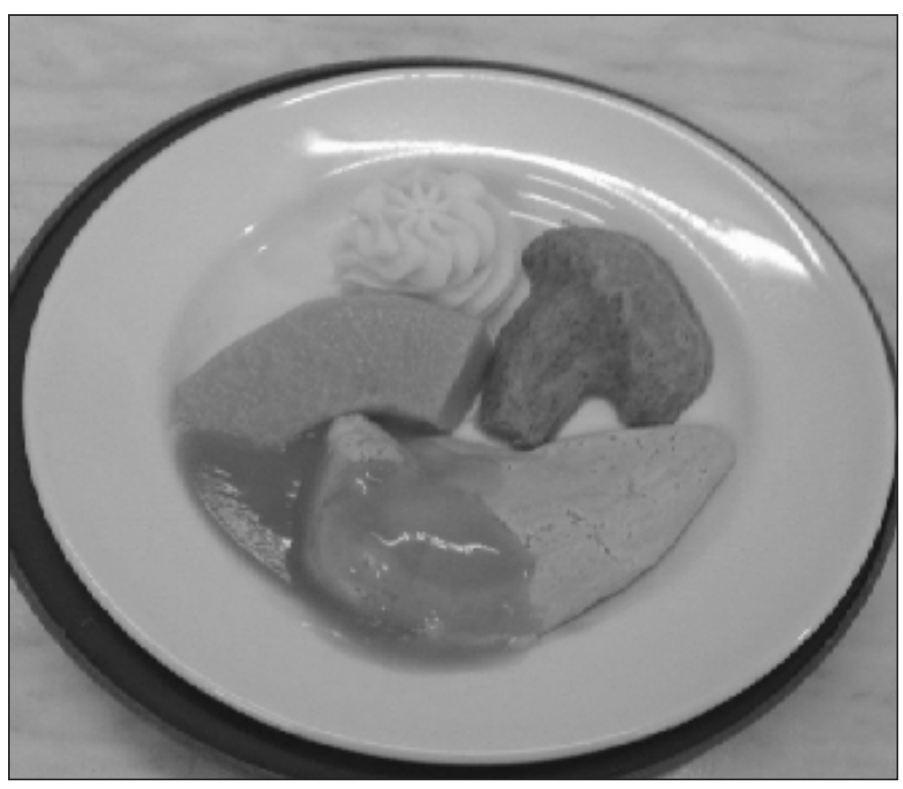

\section{Lack of access to the dining experience}

Non-participant observations found that residents, who were seated in air-comfort chairs and removed from the main dining area, were accessing the mealtime environment by sight, that is, they were watching what was happening around them and looking at the meal that was served to them.

Staff articulated a number of assumptions about providing eating assistance to residents with dysphagia and in particular those who were seated in air-comfort chairs. These included that these residents were unable to comprehend what was happening around them during mealtimes.

Care worker [1]: 'You just assume when your dropping off a vitamised meal - one, you have to feed them and two, they're not going to understand or talk to, or listen to you anyway.'

Care worker [7]: 'I wouldn't say anything purely and simply because they probably wouldn't have a clue what you were saying anyway. You know what I mean, cause they're very sick.'

\section{The lack of flavour in texture-modified food}

Another assumption articulated by care staff concerned the flavour of the non-moulded texturemodified food and that the residents with dysphagia, in particular those seated in air-comfort chairs did not require a flavoursome meal:

Field note:

Care worker giving vitamised meal to Dorothy.
Care worker [12]: 'Tastes nice?'

Resident: 'No.'

Care worker [12]: 'Oh, it does.'

Care worker [1]: 'Oh they love their salt and pepper. But I think a lot of people do and that's something we normally don't give people, like, you don't serve up a vitamised meal and say 'would you like pepper on that?', 'would you like some salt with that?' you just assume they don't.'

Enrolled Nurse [1]: 'I haven't actually tasted it. I go more on the visual, if there's a visual aspect and the food's well-presented. As long as it is well presented and the plate looks nice.'

Catering [2]: 'Well, I don't personally when I'm doing it. If I'm doing chicken rissoles, that's what I vitamise and I might put a bit of white sauce in it for the liquid. So, I vitamise the same thing anyway. So, no, I don't think it needs it (flavour enhancement).'

When asked about their opinions of the taste of the non-moulded texture-modified food three residents responded that the food was repetitive and tasteless:

Resident [5]: 'It's horrible, there's no taste and they made it taste like saw dust. They can also leave the meat, it tastes just like chalk.'

Resident [3]: 'The food's good, but it's drab.'

Investigator: 'So the food is good, but it's drab?'

Resident [3]: 'I get my grandson to buy me chutney.'

Investigator: 'So you put chutney on it? Why is it drab though?'

Resident [3]: 'It's just tasteless.'

Investigator sitting with resident in lounge room talking about vitamised food

Resident [4] seated in wheelchair looking down and shaking her head.

Resident [4]: 'It's just terrible, same old, same old.'

When residents were asked about whether nonmoulded texture-modified required more flavour they responded that flavour was a very important component to their enjoyment of their meal. Importantly, the flavour of a meal also evoked certain memories for some residents:

Investigator: 'Would you like more flavour in your vitamised meal?'

Resident [5]:' I had some mango chutney that I put in and that made a hell of a difference.'

Investigator: 'So flavour is very important to you?'

Resident [5]: 'Oh yeah, it's better as a stronger flavour.'

Resident [3]: 'That's what I like. You see the thing is I lived in India for many years and of the course the food is ahhhh [smiling]. I used to live in India you see, I had a wonderful cook who knew exactly what I liked, full of spice. I don't like it hot, but I like it spicy.'

Resident [1]: 'The spices, mmm' (smiling)

Investigator: 'The spices?'

Resident [1]: 'They're wonderful.'

Investigator: 'Is there any particular spice that you like?' 
Resident [1]: 'All of them.'

When the residents were asked whether they were involved in any food survey which elicits feedback about texture-modified food, they responded that they had never been asked for their opinions about texturemodified food.

\section{Difficulties adjusting to texture-modified food}

A key finding that emerged from the resident interviews was residents' experiences of their transition from normal food to texture-modified food. Residents stated that the transition was difficult and that eating texture-modified food required some 'getting used to':

Resident [3]: 'I don't mind either to tell you the honest truth, I got used to it now.'

Investigator: 'So you had to get used to it?'

Resident [3]: 'Yes, even now, I mean, as I say, if I didn't have some chutney or something I wouldn't eat it. I often don't.'

Resident [2]: 'It was hard. I have to get on with it because that's the way it is. I like skate (a species of fish). I can't have it - that's what I have (resident points to nonmoulded texture-modified food).'

Wife of resident [6]: 'Well, when he moved in here nearly 5 years ago he was eating normal food and then it got to the stage where he knew he was getting different food and it was hard for him. If this (moulded texturemodified food) happened twelve months ago, it would have made a difference.'

Resident [2]: 'It was sort of gradual and there's no point in being upset about it, cause that's the way it is.'

Care worker [9]: 'Maria was devastated having to go on the vitamised, yeah she used to hate it.'

\section{Moulded texture-modified food}

Non-participant observations found that there was greater interaction between care staff and residents when serving moulded texture-modified food. Care staff provided more detail about what was being served to the resident as shown in the following field notes:

Field notes:

Care worker [15] 'Here we go have some carrots, potato, chicken and broccoli and gravy of course.'

Care worker [19] 'It looks like we' re having fish today, see!'

Care worker [23] 'It's broccoli, that's it, try some broccoli.'

During the individual interviews participants responded positively toward the moulded texturemodified food. Responses included:

Care worker [12]: 'I think that looks amazing. I think that looks much better. It's a lot more appetising for the resident. The meal doesn't look as different to someone else's, it all looks the same.'
Resident [5]: 'Oh that looks better. Definitely looks more appetising than those (pointing to non-moulded texture-modified food).'

Investigator: 'What do you particularly like about it?'

Resident [5]: 'The way it's laid out. Looking at that (moulded texture-modified food), and looking at that (non-moulded texture-modified food) - no comparison.'

Care staff also described positive feelings and 'selfconscious' emotions associated with moulded texturemodified food including feeling better and emotions of pride:

Registered Nurse [1]: 'I would be quite happy with this one, even myself, it looks much better. I would be more proud to offer this food.'

Care worker [12]: 'I would feel more proud handing that over because it looks more presentable and plus I can tell what each item is.'

\section{Discussion}

The key findings of this study suggest that the mealtime experience of residents with dysphagia is fraught with a number of challenges. These include the separation of residents with dysphagia (seated in aircomfort chairs) from the dining experience, the negative influence of texture-modified food on the interaction between care staff and residents, and the difficulties experienced by residents with dysphagia as they adjust to texture-modified food.

The first key finding from the study was the identification of two very difference service styles: the service style that incorporated a number of contextualisation cues into the dining experience and the service style that demonstrated limited verbal and visual cues. Bastone's (22) analysis of the mealtime experience in an industrial setting and Gumperz's (23) notion of contextualisation cues provide insight into how the mealtime environment can affect the quality of the dining experience for both residents and staff. A contextualization cue has been defined as 'any verbal or non-verbal sign that helps speakers hint at, or clarify, and listeners to make such inferences' (23). For example, this might be the rising of the voice as a signal of encouragement to a resident following the successful swallow of a bolus of food. Non-verbal behaviors function as contextualisation cues through laughter, eye contact and touch. Importantly, there is a conceptual link between the artifacts that constitute the mealtime environment and the inferences that people make from these objects (24). Objects that may be found in the aged care mealtime environment include overway tables, clothing protectors, medication trolleys, barrack-style table arrangements and functional crockery (e.g. lipped plates) and cutlery designed for healthcare. These objects can trigger presuppositions, whereby staff retrieve from their memory the understanding of mealtimes within institutionalised spaces, such as 
a hospital. Consequently, staff act, either functionally or domestically, according to those presuppositions within the given mealtime space (22). The relationship between contextualisation cues and their influence on our social world (e.g. the social mealtime event) poses further questions concerning the influence that these contextualisation cues have on residents upon entering into a residential aged care facility that espouses a 'homelike' dining environment, but where many verbal, nonverbal and artifact contextualisation cues may present otherwise.

The second key finding of this study suggests that care staff hold certain presuppositions toward residents seated in air-comfort chairs and that these chairs (as a contextualisation cue) impose perceived limitations on residents. For example, care staffs' inability to interact normally with these residents and the inability of residents to respond normally to care staff place certain limitations on the interaction itself. Therefore, when care staff understand, and notice residents' inability to interact normally (i.e. an understanding that is made tactic through care plans and at handover) interpretive processes are taken for granted and tend to go unnoticed. Consequently, care staff do not react to any new verbal or non-verbal cues sent by the resident or care staff become unaware of the function of these new cues, interpretations may differ and misunderstandings may occur $(23,24)$. For instance, a finding of this study found that a care worker dismissed a resident's (seated in an air-comfort chair) negative response to the taste of the food. This response may be due to the care worker's presuppositions toward the resident seated in the aircomfort chair and that these presuppositions are taken for granted despite the resident's complaint. Gumperz (25) suggests that when this happens and when a difference in interpretation is brought to a person's attention, it tends to be seen in attitudinal terms. Consequently, the resident may be perceived as being 'difficult' rather than the food actually tasting bad. This then reshapes an entire course of interaction whereby the staff may misinterpret residents need for flavorsome meals. The same cues may be interpreted entirely differently by care staff when a resident is seated at a dining table and on a standard chair. The relationship between the objects that are used within institutional mealtime environments and social interaction poses important questions about the use of contextualisation cues within the mealtime environment and how changing these cues may improve the quality of the dining experience for residents and the service provided by staff.

The third key finding of this study calls for further exploration into how contextualisation cues influence our 'self-conscious' emotions. Non-moulded texturemodified food elicited negative responses toward the meal and participants also experienced difficulties describing the components of the meal (i.e. carrots, broccoli etc...). The presence of strong negative feelings and the absence of a description created a problem of interaction between care staff and residents. Importantly, when care staff articulated feelings of guilt and embarrassment at serving non-moulded texturemodified food to residents the role of care staff was compromised because they had to mitigate their negative feelings toward the meal and their inability to describe the meal with the caring value of trust held between the residents and themselves. Tangney and colleagues (26) suggest that our 'self-conscious' emotions represent a key element of our human moral apparatus, influencing the link between moral standards and moral behavior. Shame, guilt, embarrassment and pride are members of a family of 'self-conscious' emotions that are evoked by self-reflection and self-evaluation. For the care staff in this study, the 'self-conscious' emotions of guilt and embarrassment place them in a precarious position of having to find coherence between the truth (e.g. nonmoulded texture-modified food is 'crap') and articulating a falsehood by encouraging and prompting the resident to eat using positive terminology. The relationship between the aesthetic cue of food and interaction will require further qualitative investigation.

This study shows that moulded-texture-modified food improved the mealtime experience of residents with dysphagia and that preparing food that is recognisable and describable changed the way in which care staff communicated to these residents and how they felt about serving texture-modified food. However, the study demonstrates that residents' adjustment to texturemodified food was difficult and that these experiences may potentially extend to residents' families. There is limited available evidence about how older people with dysphagia adjust to texture-modified food, with the majority of research focusing on adjustments to the resident's diet to maintain or restore the safety of oral feeding (1). Further investigation is required into the nature of adjustment, highlighting the experiences and events as older people with dysphagia come to terms with their swallowing difficulties and changes to their diet.

Whilst evidence suggests that there is some decline in the taste and olfactory receptors of older people, the qualitative findings of this study has found that the flavour of texture-modified food plays an important part in the dining experience of residents with dysphagia. Further investigation is warranted into the use of flavour enhancers and aroma to improve the eating experience of residents with dysphagia. Furthermore, despite food satisfaction being crucial to the mealtime experience of residents and in ensuring adequate nutrition, neither site elicited feedback from residents about their level of satisfaction with texture-modified foods. A survey is warranted that examines the food satisfaction of people with dysphagia, specifically focusing on the domains of meal choice, food characteristics, eating assistance and mealtime experience, anxiety or worry with eating, global 
satisfaction, swallowing and chewing difficulties.

\section{Limitations}

Limitations to this study include the exploratory nature of the research and the multiple comorbidities experienced by older people in residential aged care facilities, resulting in the high study withdrawal rate from the initially planned 22 residents in this study trial to 12 residents. Care should be taken when extrapolating findings to other residential aged care facilities.

\section{Conclusion}

Residents' experience of adjustment to texturemodified food is difficult and food that is unrecognisable and indescribable creates a problem of interaction between care staff and residents during mealtimes. The implementation of moulded texture-modified food resulted in positive qualitative outcomes in the perception of texture-modified food and improved the verbal interaction between care staff and residents, indicating a more positive dining experience. Further targeted research is required into this important area of nutritional care in order to ensure that all residents are provided with nourishing, tasty and socially inclusive meals.

Conflict of interests: None

\section{References}

1. Stratton, R. J., Green, C. J. \& Elia, M. Disease-related malnutrition: an evidence-based approach to treatment, Oxfordshire, CABI Publishing, 2003.

2. Banks, M., Ash, S., Bauer, J. \& Gaskill, D. Prevalence of malnutrition in adults in Queensland public hospitals and residential aged care facilities. Nutrition and Dietetics, 2007;64, 172-178.

3. Grieger, J., Nowson, C. \& Leigh Ackland, M. Anthropometric and biochemical markers for nutritional risk among residents within an Australian residential care facility. Asian Pacific Journal of Clinical Nutrition, 2007;16, 178-186.

4. Speech Pathology Australia 2004 Position Paper: Dysphagia General, The Speech Pathology Association of Australia Limited, Victoria, Australia.

5. Dietitians Association of Australia and the Speech Pathology Association of Australia Limited. Texture-modified foods and thickened fluids as used for individuals with dysphagia: Australian standardized labels and definitions.
Nutrition \& Dietetics, 2007;vol. 64 (Suppl2), pp. S53-S76.

6. Ekberg, O., Hamdy, S., Woisard, V., Wuttge-Hannig, A., Ortega, P. Social and psychological burden of dysphagia: Its impact on diagnosis and treatment. Dysphagia, 2002;vol. 17, no. 2, pp. 139-146.

7. Kulikowski, J. Dysphagia in assisted living. Provider, 2008;vol. 34, no.12, pp. 37, 39, 41-2.

8. Keller H., Chambers L., Niezgoda, H. \& Duizeer, L. Issues associated with the use of modified texture foods. The Journal of Nutrition, Health \& Ageing, 2012;vol. 16, no. 3, pp. 195-200.

9. Douglas, M. Deciphering a meal. Dædalus, 101(1), 61-81. (Reprinted in Implicit meanings: essays in anthropology (1972;pp. 249-275). London: Routledge and Kegan Paul (1975).

10. Meiselman, H.L. Dimensions of the meal. Aspen Publishers, Inc. Maryland, 2000.

11. Creusen, M.E.H. \& Schoormans, J.P.L. The different roles of product appearance in consumer choice. Journal of Product Innovation Management, 2005; vol. 22, pp. 63-81.

12. Lawless, H.T. \& Heymann, H. Sensory Evaluation of Food: Principles and Practices, 2nd edition. Chapmann \& Hall, New York, 2010.

13. Germain, I., Dufresne, T. \& Gray-Donald, K. A Novel Dysphagia Diet Improves the Nutrient Intake of Institutionalized Elders. Journal of the American Dietetic Association, 2006;vol. 106, pp. 1614-1623.

14. Schutt, R. K. Investigating the social world (7th ed.). California, Thousand Oaks, SAGE Publications, 2011.

15. Spradley, J. P. Participant Observation, London, Wadsworth Thomson Learning, 1980.

16. Bowling, A. Research methods in health: Investigating health and health services, Buckingham, Open University Press, 2002.

17. Burns, N. \& Grove, S.K. The Practice of Nursing Research: Conduct, Critique and Utilization, Missouri, Elsevier Saunders, 2005.

18. Denzin, N. K. The Research Act - A Theoretical Introduction to Sociological Methods, New Jersey, Prentice Hall, 1989

19. QRS International 2014, Australia, viewed 2 February 2013, <http:/ / www. qsrinternational.com/ contact.aspx>

20. Spencer L., Ritchie, J. \& O'Connor, W. Analysis: Practices, Principles and Processes. IN RITCHIE, J. \& LEWIS, J. (Eds.) Qualitative Research Practice: A Guide for Social Science Students and Researchers. London, SAGE Publications, 2003.

21. Ritchie J., Spencer, L. \& O'Connor, W. Carrying out Qualitative Analysis IN RITCHIE, J. \& LEWIS, J. (Eds.) Qualitative Research Practice: A guide for Social Science Students and Researchers. London, SAGE Publications Ltd, 2003.

22. Bastone, E. The Hierarchy of Maintenance and the Maintenance of Hierarchy: Notes on Food and Industry. IN MURCOTT, A. (Ed.) The Sociology of Food and Eating. England, Gower Publishing Company Limited, 1983.

23. Gumperz, J. Contextualization and understanding. IN DURANTI, A. \& GOODWIN, C. (Eds.) Rethinking context: Language as an interactive phenomenon. Great Britain, Cambridge University Press, 1992.

24. Wilson, A. When Contextualization Cues Mislead: Misunderstanding, Mutual Knowledge, and Non-Verbal Gestures. California Linguistic Notes, 2004; vol. 29, no. 1, pp. 1-4.

25. Gumperz, J. Discourse strategies, United States of America, Cambridge University Press, 1982.

26. Tangney, J.P., Stuewig, J. \& Mashek, D.J. Moral Emotions and Moral Behavior. National Institute of Health Public Access. 2007; Vol. 57, pp. 345372.

27. Figure 2. Texture Concept Foods Pty. Ltd 